\title{
The Transport Performance of Feedback Coupled Brownian
}

\section{Ratchets}

\author{
LiMing Fan, MingTao Lv, RenZhong Huang and TianFu Gao* \\ College of Physical Science and Technology, Shenyang Normal University, Shenyang 110034, China \\ Corresponding Email: tianfugao@synu.edu.cn
}

\begin{abstract}
The directed transport properties of feedback coupled Brownian ratchets under the effect of external periodic force and load force are investigated. The influence of the coupling strength on the transport properties of coupled system is discussed in detail. It is found that the centre-of-mass mean velocity, the average diffusion coefficient and the energy conversion efficiency of the feedback system can achieve the maximum for the different optimal coupling strength, and the maximum move to right with the increase of the natural length. It implies that the optimal coupling strength and natural length can promote the directed transport and energy conversion efficiency of the feedback ratchets.
\end{abstract}

Keywords. Bioengineering, feedback-control, directed transport, energy conversion efficiency.

\section{Introduction}

Brownian motion in the environment of the thermal fluctuations is a long-study issue in nonequilibrium statistical physics. In recent years, the directed transport properties of Brownian ratchets attract the widespread attention of scholars [1, 2] Only when the systems meet the spatio-temporal symmetry-breaking feature can produce the directed transport. The Brownian ratchets satisfy this condition, and that can take advantage of noise as it converts random fluctuations into directed motion without external force [3-5] Some properties of directed transport can be obtained through this interesting phenomenon, such as the center-of-mass mean velocity, the energy conversion efficiency, etc. In addition, Brownian ratchets have the wide application in bioengineering and nanotechnology.

There are some work of the directed transport for Brownian ratchets $[6,7]$ The main concern of this paper is the directed transport properties of the feedback coupled Brownian ratchets under the external force and the effect of coupling. For discussing the transport properties of coupled ratchets in detail, the center-of-mass mean velocity, the average diffusion coefficient, the $P e$ number and the energy conversion efficiency of the coupled ratchets are investigated. It is interesting to find that there is an optimal coupling strength which can facilitate the directed transport and diffusion of the feedback system, and the energy conversion efficiency can reach a maximum as the coupling strength increasing. Meanwhile, these conclusions can provide some guidance for the enhancement of the efficiency in nano-machines.

\section{Model}


The coupled Brownian particles are acted on the asymmetric periodic ratchet potential $U\left(x_{i}\right)$, and then the particles are under the effect of asymmetric periodic force $F(t)$, load force $\lambda$, feedback control switch $\varepsilon(t)$, and the thermal noise. The dynamic behavior of particles can be described by the overdamped Langevin equation [8]:

$$
\begin{array}{r}
\gamma \dot{x}_{1}(t)=\varepsilon(t) G\left(x_{1}(t)\right)-k\left(x_{1}-x_{2}-l\right)-\lambda+F(t)+\xi_{1}(t) \\
\gamma \dot{x}_{2}(t)=\varepsilon(t) G\left(x_{2}(t)\right)+k\left(x_{1}-x_{2}-l\right)-\lambda+F(t)+\xi_{2}(t)
\end{array}
$$

The locations of the two coupled particles at the time $t$ are represented by $x_{1}(t)$ and $x_{2}(t), \gamma$ indicates the friction coefficient. $k$ is the elasticity coefficient and $l$ is the natural length. $\lambda$ denotes the load force. The external force acting on the particles is $F(t)$, and it can be expressed as follows,

$$
F(t)=\left\{\begin{array}{c}
A, \quad n \tau \leq t<n \tau+\tau_{1} \\
-A, \quad n \tau+\tau_{1} \leq t<(n+1) \tau
\end{array} .\right.
$$

In Eq. (3), $A$ is the amplitude of periodic external force, $\tau$ is the period of external force. In addition, $\xi_{1}(t)$ and $\xi_{2}(t)$ are Gaussian white noise of zero mean and $\left\langle\xi_{i}(t) \xi_{j}(t)\right\rangle=2 D \delta_{i j} \delta\left(t-t^{\prime}\right), D$ is the strength of noise. $G\left(x_{i}(t)\right)$ in Eqs. (1) and (2) denotes the force acted on the particles by the ratchet potential, and it can be given by the relation

$$
G\left(x_{i}(t)\right)=-\frac{d}{d x_{i}} U\left(x_{i}\right),
$$

where $U\left(x_{i}\right)$ indicates the external periodic potential, and can be expressed as follows,

$$
U\left(x_{i}\right)=\left\{\begin{array}{cc}
\frac{d x_{i}}{c}, & x_{i} \leq c, \\
\frac{d\left(1-x_{i}\right)}{1-c}, & c<x_{i} \leq L,
\end{array}\right.
$$

$d$ is the barrier height. $L$ is the period of ratchet potential, and the ratchet period $L=1$.

In the Eqs. (1) and (2), we consider a controller $\varepsilon(t)$, which switches the potential on $(\varepsilon=1)$ if the ensemble average of the force is positive or switches the potential off $(\varepsilon=0)$ for otherwise. And the alternative controller can be expressed as follows

$$
\varepsilon(t)=\Theta(g(t))= \begin{cases}0, & g(t) \leq 0, \\ 1, & g(t)>0,\end{cases}
$$

where $\Theta$ is Heaviside function. In Eq. (6), $g(t)$ can be expressed as

$$
g(t)=\frac{1}{2}\left(G\left(x_{1}(t)\right)+G\left(x_{2}(t)\right)\right) .
$$

So Eqs. (1) and (2) are the motion equations of the feedback coupled Brownian particles.

In order to obtain the transport properties of feedback ratchets, the center-of-mass mean velocity of the coupled particles is investigated, and it can be written as [9]

$$
<V_{c m}>=\lim _{T \rightarrow \infty} \frac{1}{2 T} \sum_{i=1}^{2} \int_{0}^{T} \dot{x}_{i}(t) d t
$$

which $<\cdot>$ indicates the ensemble average. Because of the diffusion of coupled Brownian particles at all times, we describe the fluctuation of particle displacement by introducing the average effective diffusion coefficient $D_{\text {eff }}[10]$

$$
D_{\text {eff }}=\lim _{T \rightarrow \infty} \sum_{i=1}^{2} \frac{<x_{i}(t)^{2}>-<x_{i}(t)>^{2}}{2 \cdot 2 T} .
$$

In addition, we also calculate the $P e$ number for describing the directed transport properties of the coupled Brownian particles [11]

$$
P e=\frac{|V| L}{D_{e f f}} .
$$

The $P e$ number reflects the ability of the directed transport of the feedback ratchets.

For discussing the properties of directed transport from the view of energy transform, the energy conversion efficiency of the coupled ratchets $\eta$ is calculated[12]. As usual, the efficiency $\eta$ is defined as the ratio of the useful 
work $W$ which accomplished by the system in pumping coupled particles against the load force $\lambda$ to the input of energy $P_{i n}$ from the external fluctuation. That is

$$
\eta=\frac{W}{P_{i n}}
$$

Where

$$
W=\sum_{i=1}^{2} \lambda_{i} \cdot<V_{i}>
$$

and,

$$
P_{i n}=\sum_{j=1}^{2} \frac{1}{\tau} \int_{x_{j}(n \tau)}^{x_{j}((n+1) \tau)} F_{j}(t) d x_{j}(t) .
$$

In this paper, the Eqs. (1) and (2) are numerically solved by using the stochastic Runge-Kutta algorithm. In order to obtain a stable ensemble average, we simulate $5 \times 10^{3}$ different trajectories, and each track evolves 50 periods with the time step $h=10^{-3}$. All physical quantities are used the normalization processing, no special description, the parameters take $\gamma=1.0, l=0.4, L=1.0, d=2.5, \tau=10.0, \tau_{1}=0.8, c=0.96$, and $\lambda=1.0$.

\section{Results and discussion}

Figure 1(a) shows the curves of the center-of-mass mean velocity $\left\langle V_{c m}\right\rangle$ varying with the coupling strength $K$ for different natural lengths. We find that the too weak or strong coupling strength can not facilitate the directed transport. So there is an optimal value of the coupling strength which can make the enter-of-mass mean velocity achieve the maximum. Furthermore, the optimal coupling strength moves to right with the increase of the natural length, this indicates that the feedback ratchets need the larger coupling strength to make the directed transport of coupled system reach the maximum.
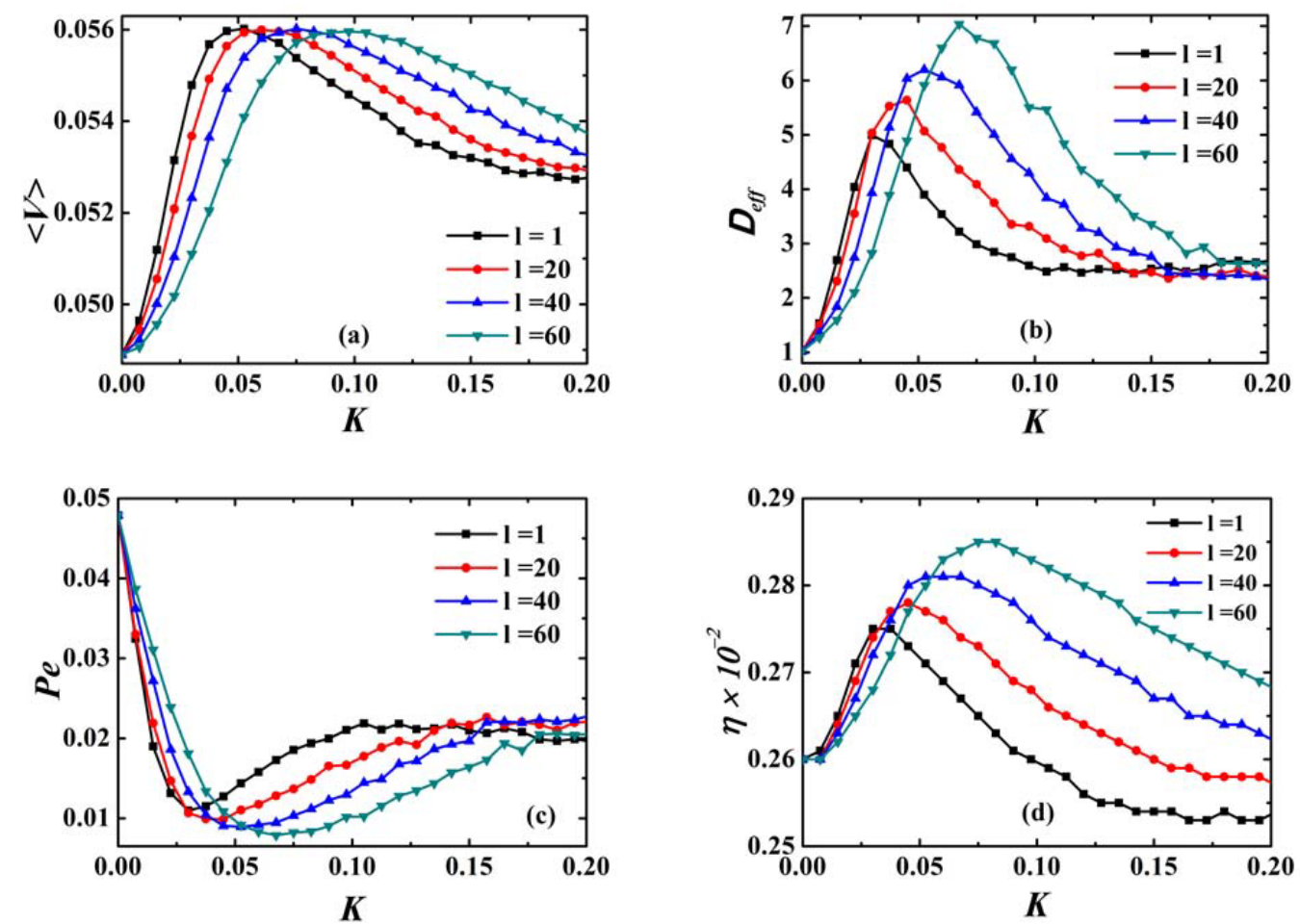

Fig. 1. The curves of (a) the center-of-mass mean velocity $\left\langle V_{c m}\right\rangle$, (b) the average effective diffusion coefficient $D_{\text {eff }}$, (c) the $P e$ number, and (d) the energy conversion efficiency $\eta$ varying with the coupling strengths $K$. 
In order to obtain the directed transport of Brownian particles in depth, the average diffusion coefficient $D_{\text {eff }}$ varying with the coupling strength for different natural lengths is discussed. The result is shown in Figure 1(b). It can be found that the average diffusion coefficient $D_{\text {eff }}$ can achieve the maximum with the increase of coupling strength, and the maximum of the coupling strength increases as the natural length increasing. In addition, we can clearly see that it is not constructive to promote the diffusion of the feedback ratchets when the coupling strength is too weak or strong. It can be explained that the weak coupling effect is not enough to cause the coupled particles diffusion. Moreover, the strong coupling effect restricts the particles and hinders the diffusion of the feedback ratchets. Furthermore, when the coupling strength is fixed, if $K=0.075$, the feedback ratchets diffuse more easily with the increase of the natural length.

Figure 1(c) shows the relation between the $P e$ number and the coupling strength for different natural lengths. From Figure 1(a) and 1(b), it is easy to observe that the curves of the diffusion coefficient achieve the maximum when the center-of-mass mean velocity reaches the maximum. Due to the diffusion occupies the dominant in the competition between the directed transport and diffusion, so according to Eq. (10), the $P e$ number reaches the minimum for the same coupling strength, as shown in Figure 1(c).

For understanding the transport property of feedback ratchets from the perspective of energy utilization, the energy conversion efficiency is discussed. As shown in Figure 1(d), it is clearly found that the efficiency curves and the velocity curves have the similar results. The efficiency of the coupled ratchets is not large when the coupling strength is too weak or strong. Therefore, the optimal coupling strength can promote the efficiency of energy conversion to reach the maximum. From Figure 1(a) and 1(d), it is interesting to note that the coupled ratchets can produce larger transport when the particles use the input energy more efficiently. And the maximum of efficiency will move to right as coupling strength increasing. It implies that the feedback ratchets need larger coupling to make the energy conversion efficiency achieve the maximum with the increase of the natural length.

\section{Conclusion}

The directed transport properties of feedback coupled Brownian ratchets under the effect of external periodic asymmetric force and load force are investigated, and the impact of the coupling strength on the transport properties of coupled ratchets is discussed in detail. It is found that the center-of-mass mean velocity, the average diffusion coefficient, and the energy conversion efficiency can achieve the maximum as the coupling strength increasing for the different natural lengths. In addition, the $P e$ number reaches the minimum with the increase of coupling strength. Therefore, the optimal parameters are important to enhance the directed transport of feedback coupled ratchets. These conclusions can be applied in separation of particles and the improvement of the efficiency for biomolecular motors.

\section{Acknowledgement}

This work is supported by the National Natural Science Foundation of China (Grant No. 11347003) and the Excellent Talents Program of Shenyang Normal University (Grant No. 91400114005).

\section{References}

1. L. Angelani, A. Costanzo, R. Di Leonardo, EPL 96, 68002 (2011).

2. P. K. Ghosh, V. R. Misko, F. Marchesoni, F. Nori, Phys. Rev. Lett. 110, 268301 (2013).

3. J. E. Villegas, S. Savel'ev, F. Nori, E. M. Gonz'alez, J. V. Anguita, R. Garc'1a, J. L. Vicent, Science 302, 1188 (2003). 
4. M. Salerno, Y. Zolotaryuk, Phys. Rev. E 65, 056603 (2002).

5. S. Ooi, S. Savel'ev, M. B. Gaifullin, T. Mochiku, K. Hirata, F. Nori, Phys. Rev. Lett. 99, 207003 (2007).

6. $\quad$ Ai B Q, Wu J C, J. Chem. Phys. 140, 094103 (2014).

7. W. E. Uspal, M. N. Popescu, S. Dietrich, M. Tasinkevych, Soft Matter 11, 434 (2015).

8. Li G, Tu Z C, Sci. China-Phys. Mech. Astron. 59, 640501 (2016).

9. Zheng Z G, Cross M C, Hu G Phys. Rev. Lett. 89, 154102 (2002).

10. Wang L F, Gao T F, Huang R Z, Zheng Y X, Acta. Phys. Sin. 62, 070502 (2013).

11. Gao T F, Chen J C, J. Phys. A: Math. Theor. 42, 065002 (2009).

12. Sumithra K, Sintes T, Physica A 297, 112 (2001). 